\title{
Effects of Single Prolonged Stress and D-Cycloserine on Contextual Fear Extinction and Hippocampal NMDA Receptor Expression in a Rat Model of PTSD
}

\author{
Shigeto Yamamoto', Shigeru Morinobu', Manabu Fuchikami', Akiko Kurata', Toshiro Kozuru' and \\ Shigeto Yamawaki*, \\ 'Department of psychiatry and Neurosciences, Division of Frontier Medical Science, Programs for biomedical Research, Graduate School \\ of Biomedical Sciences, Hiroshima University, Minami-ku, Hiroshima, Japan
}

\begin{abstract}
Although the impaired extinction of traumatic memory is one of the hallmark symptoms of posttraumatic stress disorder (PTSD), the underlying mechanisms of impaired extinction are unclear and effective pharmacological interventions have not yet been developed. Single prolonged stress (SPS) has been proposed as an animal model of PTSD, since rats subjected to SPS (SPS rats) show enhanced negative feedback of the HPA axis and increased contextual fear, which are characteristics similar to those observed in patients with PTSD. In this study, using SPS rats, we examined (a) the ability of SPS to impair fear extinction, (b) whether D-cycloserine (DCS) can alleviate impaired fear extinction in SPS rats, and (c) the effect of SPS and/or DCS on the levels of N-methyl-D-aspartate (NMDA) receptor subunit mRNAs in the rat hippocampus during extinction training. SPS rats exhibited impaired fear extinction in the contextual fear test, which was alleviated by the repeated administration of DCS. The effect of enhanced extinction, induced by the administration of DCS to SPS rats, was maintained for one week following extinction training. SPS induced significant upregulation of the levels of NMDA receptor subunit mRNAs before and during the period of extinction training, while repeated administration of DCS eliminated the enhanced mRNA levels of NMDARs. Behavioral analyses indicated that SPS is an appropriate animal model of PTSD and that DCS may be effective in the treatment of PTSD. These findings suggest that DCS, irrespective of its mechanistic involvement in the enhancement of fear extinction, may help to reverse hippocampal plasticity, and thus reverse the NMDA compensatory alterations.

Neuropsychopharmacology (2008) 33, 2108-21 16; doi:I0.1038/sj.npp. I 301605; published online 24 October 2007
\end{abstract}

Keywords: posttraumatic stress disorder (PTSD); single prolonged stress (SPS); extinction; contextual fear conditioning; NMDA receptor subunits (NMDARs); D-cycloserine (DCS)

\section{INTRODUCTION}

Posttraumatic stress disorder (PTSD), an anxiety disorder is induced by exposure to life-threatening trauma. According to the DSM-IV diagnostic criteria, the characteristic features of PTSD include persistent reexperiencing trauma, avoidance and numbing, and hyperarousal (American Psychiatric Association, 1994). The majority of patients with PTSD exhibit long-lasting reexperience of traumatic events and subsequently avoid the stimuli that link traumatic events, even though they recognize that the traumatic event is no longer occurring. Recent advances in our understanding of the mechanisms underlying fear extinction has led to the hypothesis that dysfunctional fear extinction plays an

*Correspondence: Dr S Yamawaki, Department of Psychiatry and Neurosciences, Division of Frontier Medical Science, Programs for biomedical Research, Graduate School of Biomedical Sciences, Hiroshima University, I-2-3 Kasumi, Minami-ku, Hiroshima 734-855I, Japan, Tel: +81 82257 5205, Fax: + 81822575209 ,

E-mail: yamawaki@hiroshima-u.ac.jp

Received 9 March 2007; accepted 2 I September 2007 important role in the development of clinical symptoms such as reexperiencing of trauma in PTSD, since extinction is defined as a reduction in conditioned fear response when the conditioned stimulus is repeatedly presented in the absence of an unconditioned stimulus (Milad et al, 2006; Quirk et al, 2006; Rauch et al, 2006; Rothbaum and Davis, 2003).

Although the neurocircuitry mechanism of contextual fear extinction is not fully understood, brain regions such as the hippocampus, amygdala, and prefrontal cortex are involved in the memory extinction (Sotres-Bayon et al, 2004, 2006). Likewise, though the molecular mechanism of contextual fear extinction remains to be precisely determined, the $N$-methyl-D-aspartate (NMDA) receptor appears to play a critical role in fear extinction (Falls et al, 1992; Ledgerwood et al, 2003, 2004, 2005; Rodrigues et al, 2001; Santini et al, 2001; Walker et al, 2002). In addition to amygdaloid NMDA receptors, emerging evidence has recently indicated that hippocampal NMDA receptors (NMDARs) and subsequent signaling pathways are involved in the mechanism for extinction of contextual fear 
(Bevilaqua et al, 2005; Szapiro et al, 2003). For example, Szapiro et al (2003), using a step-down avoidance paradigm, found that intrahippocampal infusions (CA1 region) of the NMDA receptor antagonist, AP5, immediately after the first of four daily extinction exposures, produced a lasting impairment that persisted even with additional extinction training without infusions.

With regard to facilitatory effects of pharmacological agents on fear extinction, recent studies with rats have indicated that the extinction of particular fear responses, such as freezing (Ledgerwood et al, 2003, 2004, 2005) and fear potentiated startle (Walker et al, 2002), can be enhanced with the glycine partial agonist D-cycloserine (DCS). DCS enhances excitatory neurotransmission mediated by NMDA receptors, by binding to the strychnine-insensitive glycine recognition site of the NMDA receptor complex, without inducing neurotoxicity. Indeed, in clinical studies, Ressler et al (2004) and Hofmann et al (2006) have recently reported that DCS facilitates fear extinction in patients with anxiety disorders.

On the other hand, development of not only an effective treatment but also appropriate animal model will promote our understanding of mechanisms of PTSD. For instance, Liberzon et al $(1997,1999)$ proposed a rat model of PTSD involving single prolonged stress (SPS). Rats subjected to SPS (SPS rats) show enhanced negative feedback of the hypothalamic-pituitary-adrenal (HPA) axis function in response to glucocorticoid administration (Liberzon et al, 1997, 1999). In addition, SPS rats also exhibit a sustained exaggeration of the acoustic startle response (Khan and Liberzon, 2004). Furthermore, we recently demonstrated that SPS rats exhibit enhanced contextual freezing, increased anxiety-like behavior in the elevated plus maze, and stress-induced analgesia compared to rats subjected to Sham treatment (Imanaka et al, 2006; Takahashi et al, 2006). Since these behavioral responses seen in rats subjected to SPS resemble the clinical symptoms seen in patients with PTSD, it is postulated that SPS is an appropriate animal model of PTSD.

In this context, we first examined whether dysfunctional extinction of contextual fear was found in SPS rats. This would allow us to elucidate the involvement of dysfunctional extinction in the pathophysiology of PTSD. Second, we examined the effect of DCS on the dysfunctional extinction of contextual fear. We then examined alterations in the levels of mRNA coding for NMDA receptor subunits, including NR1, NR2A, NR2B, NR2C, in the hippocampus during extinction training, and the effects of DCS on NMDAR mRNA levels in the hippocampus during extinction training.

\section{MATERIALS AND METHODS}

\section{Animals}

Male Sprague-Dawley rats weighing between 300 and $350 \mathrm{~g}$ (Japan Charles River, Yokohama, Japan) were used in our studies. The animals were group-housed (three per cage) and maintained on a 12-h light/dark cycle with food and water freely available. All procedures took place during the light cycle. A total of 176 rats were used in this study (Experiment 1: $N=20$, Experiment 2: $N=76$, Experiment 3:
$N=44$, Experiment 4: $N=36$ ). All animal procedures were conducted in strict accordance with the Hiroshima University School of Medicine Animal Care Committee Guiding Principles on Animal Experimentations in Research Facilities for Laboratory Animal Science, School of Medicine, Hiroshima University.

\section{Single Prolonged Stress (SPS)}

According to the method by Liberzon et al (1997, 1999), SPS was conducted in three stages: restraint for $2 \mathrm{~h}$, forced swim for $20 \mathrm{~min}$, and ether anesthesia. Each rat was restrained for $2 \mathrm{~h}$ by placing it inside a disposable clear polyethylene cone bag (Asahikasei, Tokyo, Japan) with only the tail protruding (Suenaga et al, 2004). The large end of the cone was closed with tape at the base of the tail. The bag size was adjusted according to the size of the rat in order to achieve complete immobilization. A hole in the small end of the cone allowed the rats to breathe freely. After immobilization, they were individually placed in a clear acrylic cylinder $(240 \mathrm{~mm}$ diameter, $500 \mathrm{~mm}$ height), filled two-thirds from the bottom with water $\left(24^{\circ} \mathrm{C}\right)$ and forced to swim for $20 \mathrm{~min}$. Following 15 min recuperation, they were exposed to diethyl ether until loss of consciousness, and then left undisturbed in their home cage for 7 days.

\section{Contextual Fear Conditioning and Extinction Training}

In the first experiment, we investigated influence of SPS on fear extinction (Experiment 1). Animals were randomly assigned to two groups (SPS, Sham). Sham rats were left alone without handling in their cages. During SPS treatment, Sham rats were moved and placed in an identical cage where SPS was conducted. Rats were placed in a conditioning chamber $(325$ width $\times 280$ height $\times 500$ depth $\mathrm{mm}$ ), and then were exposed to a 180-s conditioning context without any stimulation (ie a tone). Immediately after that, they received a $4-\mathrm{s}, 0.8-\mathrm{mA}$ footshock through a stainless steel grid floor by a shock generator-scrambler (SGS-003: Muromachi, Tokyo, Japan). Two footshocks were delivered with an intertrial interval of $30 \mathrm{~s}$. Following the footshock, rats remained in the chamber for an additional $1 \mathrm{~min}$ before being returned to their home cages.

Extinction training was defined as the repetitive exposure to the contextual cue (the apparatus) in the absence of footshock. Twenty-four hours after fear conditioning, rats were placed for $10 \mathrm{~min}$ without footshock in the same chamber where the footshock was delivered. In a similar manner, extinction training was performed on each of five consecutive days following fear conditioning (Figure 1). Freezing was monitored using a time sampling method in which each rat was observed once every $5 \mathrm{~s}$ and a percentage score was calculated for the proportion of the total observation period spent freezing. Freezing was defined as the total absence of body or head movement except for that associated with breathing. Freezing behavior of the rat was recorded on videotape, and later scored blindly by welltrained experimenters. Pearson's correlation coefficient was calculated to examine the interrater reliability. The interrater reliability between the two scorers was high $(r=0.96)$. 


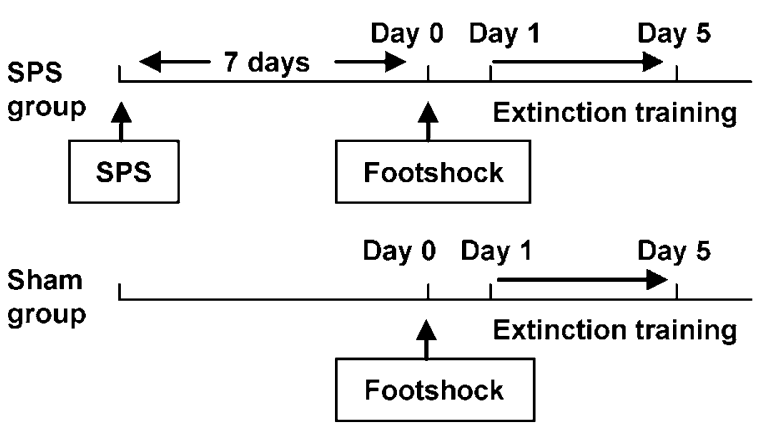

Figure I Treatment groups and procedure. In the SPS group, 7 days after SPS treatment, contextual fear conditioning was performed (on day 0 ), and then extinction training was performed on each of five consecutive days following fear conditioning. In the Sham group, the procedures were similar except that SPS treatment was not performed.

\section{Drug Treatment}

In this experiment, we investigated the effects of DCS on fear extinction. DCS was purchased from Sigma-Aldrich (St Louis, MO, USA). To avoid stress from the injection per se and to mimic clinical use, we administered DCS orally. For DCS administration, DCS was administered daily for 6 days (from the end of fear conditioning to the beginning of the fifth extinction training) (Experiment 2A).

Animals were randomly assigned to four groups (SPS, SPS + DCS, Sham, Sham + DCS). In addition, to examine the sustained effect of DCS upon fear extinction, we evaluated the freezing responses of animals from each of the four groups one week after the fifth extinction training period, in the absence of DCS. The two groups treated with DCS (SPS + DCS, Sham + DCS) were not treated with DCS between the fifth extinction training period and day 12 (Experiment 2B). Milli-Q water was available freely for the SPS and Sham groups, and DCS dissolved in Milli-Q water was available freely for the SPS + DCS and Sham + DCS groups. The DCS dose $(15 \mathrm{mg} / \mathrm{kg}$ per rat $)$ was chosen on the basis of the results of other behavioral studies (Ledgerwood et al, 2003, 2005; Walker et al, 2002). Before the present study, we measured the consumption of drinking water in our animal facility. Specifically, we measured the reduction of water volume in the animal's water bottles each day for six consecutive days in order to verify the intake of DCS. Based upon this measurement, we found that three rats (BW of each rat was approximately $330 \mathrm{mg}$ at the beginning of measurement) in one cage consumed approximately $120 \mathrm{ml}$ of Milli-Q water per day $(N=54)$. Thus, the average weight of a rat $(330 \mathrm{mg})$, was used to calculate the appropriate dose of DCS $(15 \mathrm{mg} / \mathrm{kg}$ $\times$ average weight of 3 rats $\times 6$ ). The dose was dissolved in $240 \mathrm{ml}$ of Milli-Q water. DCS solution $(240 \mathrm{ml})$ was added to the water bottle in each cage at the beginning of DCS administration (Supplementary Figure 1). During the present study, we also measured the weight of each rat daily and adjusted the concentration of DCS to $15 \mathrm{mg} / \mathrm{kg}$ per rat each day, based upon the average weight of three rats in one cage. We also verified that the three rats in any one cage consumed approximately $120 \mathrm{ml}$ of Milli-Q water, with or without DCS. Fear conditioning and extinction training were performed as described above.
Measurement of the Levels of NMDARs in the Hippocampus by Real-Time Quantitative Polymerase Chain Reaction (RT-PCR)

To elucidate whether the hippocampal glutamatergic systems are involved in fear extinction, we used RT-PCR to measure alterations in NMDAR mRNA levels in the hippocampus during extinction training (Experiment 3). Animals were randomly assigned to two groups (SPS, Sham), and were sacrificed by decapitation at the indicated time on day 0 (before fear conditioning), on day 1 (after the first context exposure), or on day 4 (after the fourth context exposure). NMDAR mRNA levels were analyzed between the two groups and then compared across experimental time points (days). Further, we investigated the effects of repeated DCS administration on the NMDAR mRNA levels in the hippocampus during extinction training (Experiment 4). DCS was repeatedly administered for 5 days (from the end of fear conditioning to the beginning of the fourth extinction training). Animals were randomly assigned to four groups (SPS, Sham, SPS + DCS and Sham + DCS), and were sacrificed by decapitation after the fourth context exposure. Hippocampal tissue was removed from the brain and quickly frozen using powdered dry ice and stored at $-80^{\circ} \mathrm{C}$. Total RNA was extracted using RNAqueous Total RNA Isolation kits (Ambion, Austin, TX, USA) according to the manufacturer's instructions, and then a single-stranded cDNA was synthesized using the QuantiTect Reverse Transcription Kit (QIAGEN, Hilden, Germany), which provided a procedure for genomic DNA elimination and reverse transcription. RTPCR was performed with an ABI7700 sequence detection system (PE Applied Biosystems, Foster City, CA, USA) to quantify relative mRNA levels in samples. RT-PCR was performed to amplify the mRNA of NMDARs. The primers and TaqMan hybridization probes were designed using Primer Express software (PE Applied Biosystems). Table 1 shows the sequences and fluorescent dyes of the PCR primers and TaqMan probes for each molecule. The TaqMan probe, which was designed to hybridize to the PCR products, was labeled with a fluorescent reporter dye at the $5^{\prime}$ end and a quenching dye at the $3^{\prime}$ end. PCR was carried out with TaqMan Universal PCR Master Mix (PE Applied Biosystems). All standards and samples were assayed in triplicate. Thermal cycling was initiated with an initial denaturation at $50^{\circ} \mathrm{C}$ for $2 \mathrm{~min}$ and $95^{\circ} \mathrm{C}$ for $10 \mathrm{~min}$. After this initial step, 40 cycles of PCR were performed. Each PCR cycle consisted of heating at $95^{\circ} \mathrm{C}$ for $15 \mathrm{~s}$ for melting and $60^{\circ} \mathrm{C}$ for $1 \mathrm{~min}$ for annealing and extension. The PCR assay for glyceraldehyde-3-phosphate dehydrogenase (GAPDH) was performed using the TaqMan Rodent GAPDH Control Reagents kit (PE Applied Biosystems). The mRNA levels of NMDARs were detected by RT-PCR (ABI PRISM 7700 sequence detection system) and the ratio of the concentration of the target molecule to that of GAPDH (target molecule/GAPDH) in unknown samples was calculated. For reference, the RT-PCR standard curve plot and the amplification plot of NR1 before fear conditioning are shown (Supplementary Figures 2 and 3).

\section{Statistical Analysis}

All values shown represent the mean \pm SEM. In Experiment 1 , freezing responses of the Sham and SPS groups were 
Table I Sequences and Fluorescent Dye of PCR Primers and TaqMan Probes

NRI

Forward primer

Reverse primer

TaqMan probe

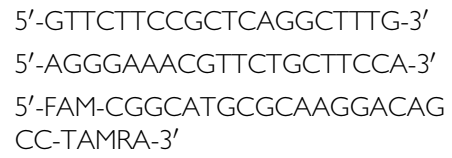

NR2A

Forward primer

5'-AGCCCCCTTCGTCATCGTA-3'

Reverse primer

5'-GACAGGGCACCGTGTTCCT-3'

TaqMan probe

$5^{\prime}-\mathrm{FAM}-$

AGGACATAGACCCCCTGACTGAGACCTG TG-TAMRA-3'

\section{$N R 2 B$}

Forward primer

Reverse primer

TaqMan probe

NR2C

Forward primer

Reverse primer

TaqMan probe

\section{5'-CCCCCAAGTTCTGGTTGGT-3' \\ 5'-TाTTGGGACGAGCTाTGCT-3' \\ 5'-FAM-TTGGCCGTCTTGGCCGTATCAGG- TAMRA-3'}

compared by two-way ANOVA (stress, day) for repeated measures (day). In order to further evaluate effects of SPS on each experimental day, we used the unpaired Student's $t$-test. In Experiment 2, freezing responses of the four groups were compared by three-way ANOVA (ie stress, drug, day) for repeated measures (day). In order to evaluate the effect of DCS upon the consolidation of original fear in Experiment 2A, we used two-way ANOVA (stress, drug) to analyze the freezing responses of animals from the four groups on day 1. In Experiment 3, mRNA levels were analyzed using the unpaired Student's $t$-test or by two-way ANOVA (stress, day) followed by appropriate post hoc comparisons. Post hoc comparisons were performed using Tukey's test. In Experiment 4, data for mRNA levels were analyzed by two-way ANOVA (stress, drug). Results were considered statistically significant at $p<0.05$.

\section{RESULTS}

\section{Experiment 1: Influence of SPS on Fear Extinction}

In Experiment 1, two-way repeated measures ANOVA showed a significant main effect of day $(\mathrm{F}(4,72)=34.01$, $p<0.01)$, stress $(\mathrm{F}(1,18)=20.14, p<0.01)$, and an interaction between stress and day $(\mathrm{F}(4,72)=2.77, p<0.05)$. In order to further evaluate the effects of SPS on each experimental day, we used the unpaired Student's $t$-test. The freezing responses on day 1 did not differ between the SPS and Sham groups $(t(18)=1.54, p=0.13)$, suggesting that contextual fear conditioning was successful in both groups of rats. During repeated context exposure, however,

a significant difference in the freezing responses between the two groups appeared on day 2, and significant differences persisted until day 5 (day 2: $t(18)=2.76$; $p<0.05$, day 3: $t(18)=3.67 ; p<0.01$, day $4: t(18)=3.63$; $p<0.01$, day 5: $t(18)=4.11 ; p<0.01$ ) (Figure 2).

\section{Experiment 2: Effects of DCS Administration on Fear Extinction}

In Experiment 2A, three-way repeated measures ANOVA demonstrated significant main effects of day $(\mathrm{F}(4,72)=105.39, \quad p<0.01), \quad$ stress $\quad(\mathrm{F}(1,18)=16.41$, $p<0.01)$, drug $(\mathrm{F}(1,18)=9.81, p<0.01)$, and significant interactions between stress and drug $(\mathrm{F}(1,18)=5.71$, $p<0.05)$, and between stress and day $(\mathrm{F}(4,72)=2.71$, $p<0.05)$. There were no significant interactions between stress and drug and day $(\mathrm{F}(4,72)=1.26, p=0.29)$, or between drug and day $(\mathrm{F}(4,72)=1.02, p=0.39)$ (Figure 3 ). In order to evaluate the effect of DCS upon the consolidation of original fear, we used two-way ANOVA to analyze the freezing responses of animals from each of the four groups on day 1. Two-way ANOVA revealed no significant effects associated with stress $(\mathrm{F}(1,36)=1.91 .1, p=0.17)$ or drug $(\mathrm{F}(1,36)=1.93 .1, p=0.17)$, and revealed no significant interaction between stress and drug $(\mathrm{F}(1,36)=0.29 .1$, $p=0.59)$. Collectively, these data suggest that DCS did not affect the consolidation of original fear.

In Experiment 2B, we examined the sustained effect of DCS upon enhanced extinction. In this experiment, rats were not treated with DCS between the fifth period of extinction training and day 12 . Both of the groups administered with DCS exhibited less freezing on day 12 as compared to day 5. Three-way repeated measures ANOVA revealed significant main effects associated with day $(\mathrm{F}(1,16)=20.71, p<0.01)$, stress $(\mathrm{F}(1,16)=21.39$, $p<0.01)$ and drug $(\mathrm{F}(1,16)=15.6, p<0.01)$. Further analysis revealed significant interactions between stress and drug $(\mathrm{F}(1,16)=7.43, p<0.05)$, between stress and day $(\mathrm{F}(1,16)=9.42, \quad p<0.01)$, and between drug and day $(\mathrm{F}(1,16)=11.92, p<0.01)$. There was no significant interaction between stress and drug and day $(\mathrm{F}(1,16)=2.66$, $p=0.12$ ) (Figure 4).

\section{Experiment 3: Measurement of the mRNA Levels of NMDARs in the Hippocampus During Extinction Training}

In Experiment 3, a total of 44 rats were used (day 0: Sham; $N=6$, SPS; $N=6$, day 1: Sham; $N=7$, SPS; $N=7$, day 4 : Sham; $N=9$, SPS; $N=9$ ). Rats showed freezing responses similar to comparable rats in Experiment 1. This was despite the fact that different sets of rats were used for each experiment.

For comparison, when we compared the SPS and Sham groups, before fear conditioning, we observed that the levels of all NMDAR subunit mRNAs in the SPS group were higher than those in the Sham group (NR 1: $t(10)=5.04 ; p<0.01$, NR2A: $t(10)=2.93 ; p<0.05$, NR2B: $t(10)=3.68 ; p<0.01$, NR2C: $t(10)=5.11 ; p<0.01)$.

The results after the first context exposure were similar to the results observed before fear conditioning; ie the levels of all NMDAR subunit mRNAs in the SPS group were also 


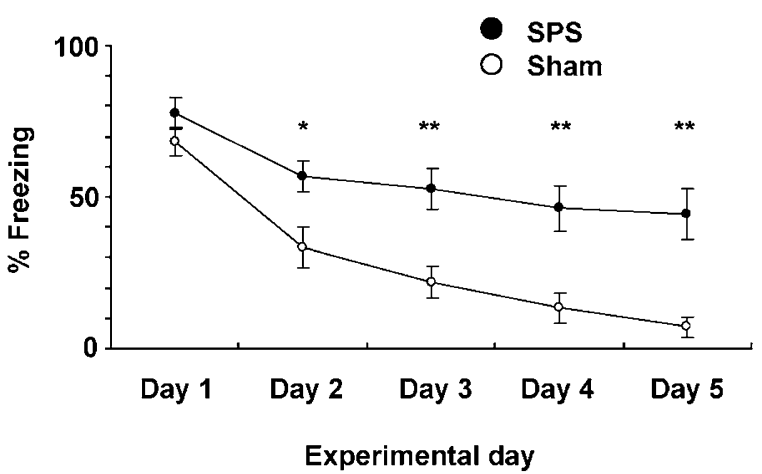

Figure 2 Influence of SPS on fear extinction. Data are expressed as mean \pm SEM of 10 rats per group. On day I, there were no significant differences in the freezing levels between the Sham and SPS groups. However, on days 2-5, the SPS group showed impaired extinction compared to the Sham group. ${ }^{*} p<0.05,{ }^{*} * p<0.0$ l; unpaired Student's $t$-test.

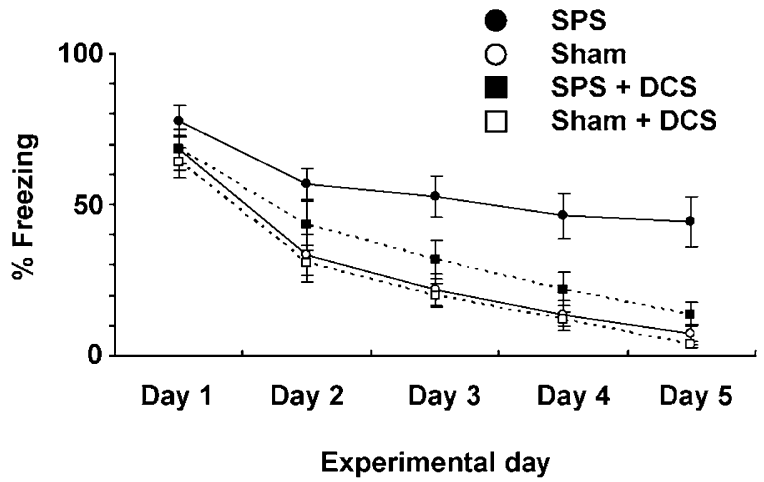

Figure 3 Effects of repeated DCS administration on fear extinction. Data are expressed as mean \pm SEM of 10 rats per group.

higher than those in the Sham group (NR 1: $t(12)=3.27$; $p<0.01$, NR2A: $t(12)=3.92 ; p<0.01$, NR2B: $t(12)=3.95$; $p<0.01$, NR2C: $t(12)=4.20 ; p<0.01)$.

After the fourth context exposure, the levels of all NMDAR subunit mRNAs in the SPS group were still higher than those in the Sham group (NR 1: $t(16)=4.81 ; p<0.01$, NR2A: $t(16)=2.97 ; p<0.01$, NR2B: $t(16)=3.53 ; p<0.01$, NR2C: $t(16)=5.63 ; p<0.01)$.

When using two-way ANOVA to compare the levels of NMDAR mRNAs across the experimental timepoints, we revealed significant effects of stress $(\mathrm{F}(1,38)=56.73$, $p<0.01)$ and day $(\mathrm{F}(2,38)=41.56, p<0.01)$ upon NR1 mRNA levels. We found no significant interaction between stress and day $(\mathrm{F}(2,38)=1.23, p=0.3)$. Post hoc analysis across experimental timepoints, revealed that there were significant differences between day 0 and day 1 , and between day 0 and day $4 \quad(p<0.01$, respectively) (Figure 5a). For NR2A mRNA levels, two-way ANOVA detected significant effects of stress $(\mathrm{F}(1,38)=22.18$, $p<0.01)$ and day $(\mathrm{F}(2,38)=7.78, p<0.01)$. No significant interaction between stress and day was found $(\mathrm{F}(2,38)=1.2, p=0.31)$. Post hoc analysis revealed that there was a significant difference between day 0 and day 1 $(p<0.01)$ when comparing experimental timepoints (Figure 5b). For NR2B mRNA levels, two-way ANOVA detected significant effects of stress $(\mathrm{F}(1,38)=35.39$,

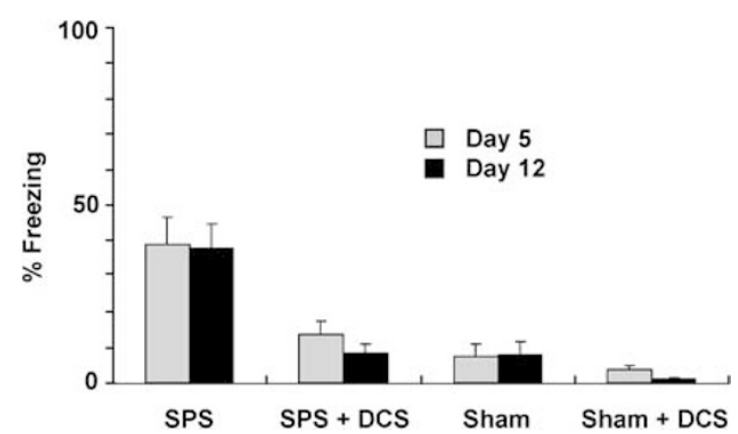

Figure 4 Effects of repeated DCS administration upon fear extinction a week after extinction training. Data are expressed as mean \pm SEM of nine rats per group.

$p<0.01)$ and day $(\mathrm{F}(2,38)=6.45, p<0.01)$. No significant interaction was found between stress and day $(\mathrm{F}(2,38)=0.39, p=0.67)$. Post hoc analysis revealed that there was a significant difference between day 0 and day 1 $(p<0.01)$ across experimental timepoints (Figure 5c). For NR2C mRNA levels, two-way ANOVA detected significant effects of stress $(\mathrm{F}(1,38)=74.38, p<0.01)$ and day $(\mathrm{F}(2,38)=22.07, p<0.01)$. No significant interaction was detected between stress and day $(\mathrm{F}(2,38)=2.12, p=0.13)$. Post hoc analysis across experimental timepoints revealed that there were significant differences between day 0 and day 1 , and between day 0 and day $4(p<0.01$, respectively) (Figure 5d).

Collectively, our comparison of NMDAR expression in the hippocampus between the SPS and Sham groups demonstrated that the levels of all four NMDAR subunits in the SPS group were significantly higher than those in the Sham group, both before and during the entire period of extinction training. In addition, in comparing data across the experimental timepoints, we have demonstrated that extinction training affected the levels of all NMDAR subunit mRNAs in the hippocampus.

\section{Experiment 4: Effects of Repeated DCS Administration on NMDAR mRNA Levels in the Hippocampus During Extinction Training}

In Experiment 4, a total of 36 rats were used $(N=9$ per group). Rats showed freezing responses similarly to comparable rats in Experiment 2A. This was despite independent sets of rats being used for each experiment.

In the analysis of NR1 mRNA levels, two-way ANOVA showed a significant effect of stress $(\mathrm{F}(1,32)=9.33$, $p<0.01)$, and a significant interaction between stress and drug $(\mathrm{F}(1,32)=8.47, p<0.01)$. No significant effect of drug was found $(\mathrm{F}(1,32)=1.22, p=0.27)$ (Figure $6 \mathrm{a})$. For NR2A mRNA levels, two-way ANOVA showed a significant effect of drug $(\mathrm{F}(1,32)=7.34, p<0.05)$ and a significant interaction between stress and drug $(\mathrm{F}(1,32)=10.4, p<0.05)$. No significant effect of stress was found $(\mathrm{F}(1,32)=2.33$, $p=0.13$ ) (Figure 6b). For NR2B mRNA levels, two-way ANOVA showed a significant effect of drug $(\mathrm{F}(1,32)=31.91, p<0.01)$ and a significant interaction between stress and drug $(\mathrm{F}(1,32)=6.11, p<0.05)$. No significant effect of stress was found $(\mathrm{F}(1,32)=3.71$, $p=0.06$ ) (Figure 6c). For NR2C mRNA levels, two-way 

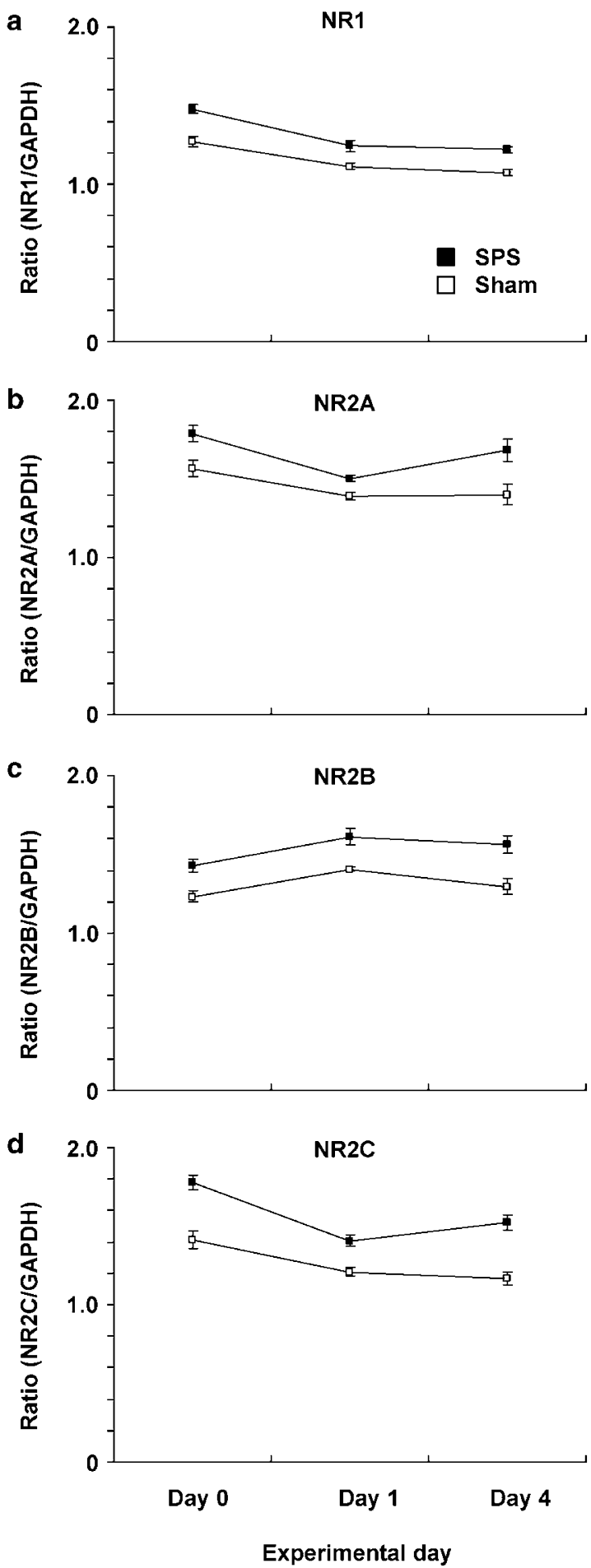

Figure 5 Comparison of the levels of NMDAR mRNAs (NRI (a), NR2A (b), NR2B (c), NR2C (d)) across experimental timepoints. Data are expressed as the ratio of the concentration of the target molecule to that of GAPDH (target molecule/GAPDH) and represent the mean \pm SEM (day 0 : Sham; $N=6$, SPS; $N=6$, day I: Sham; $N=7$, SPS; $N=7$, day 4: Sham; $N=9$, SPS; $N=9$ ).

ANOVA showed a significant effect of stress $(\mathrm{F}(1,32)=5.76$, $p<0.05)$ and a significant interaction between stress and drug $(\mathrm{F}(1,32)=12.1, p<0.01)$. No significant effect of drug was found $(\mathrm{F}(1,32)=2.36, p=0.13)$ (Figure $6 \mathrm{~d})$.
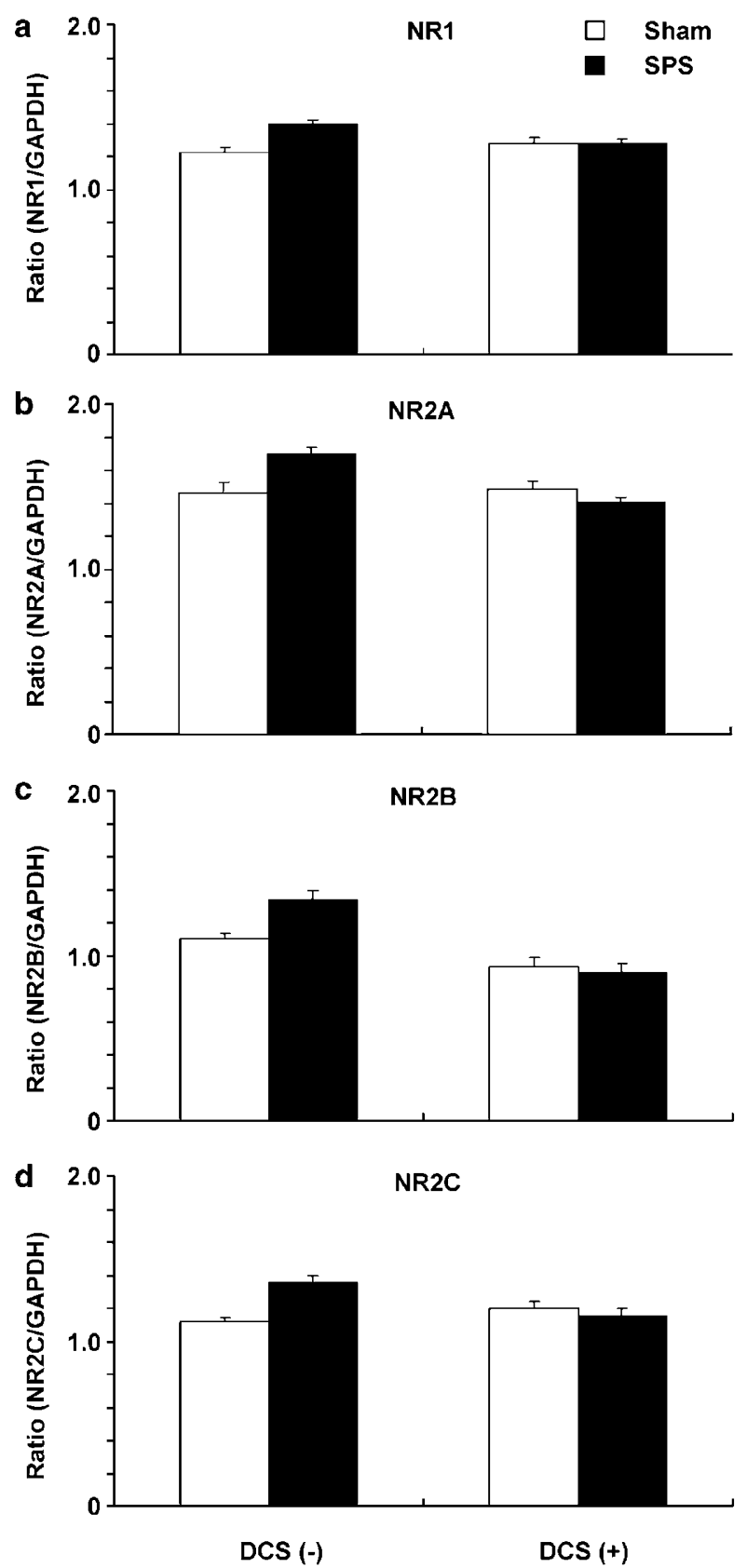

Figure 6 Effects of repeated DCS administration on the levels of NMDAR mRNAs (NRI (a), NR2A (b), NR2B (c), NR2C (d)) on day 4. Data are expressed as the ratio of the concentration of the target molecule to that of GAPDH (target molecule/GAPDH) and represent the mean \pm SEM of nine rats per group.

Collectively, the statistical analyses revealed that DCS led to a reduction of mRNA levels that was most consistent for the SPS group.

\section{DISCUSSION}

Using a contextual fear-conditioning paradigm in rats, we examined the influence of SPS on fear extinction, and the effects of DCS on the impaired fear extinction induced by SPS. We then examined alterations in the levels of NMDAR 
mRNAs in the hippocampus during extinction training and the effects of DCS on NMDAR mRNA levels in the hippocampus during extinction training. The principal findings of this study are that (1) SPS induced marked impairment of contextual fear extinction in the rats, (2) repeated administration of DCS alleviated impaired fear extinction in SPS rats, and enhanced extinction induced by the administration of DCS was maintained for one week after extinction training, (3) the levels of mRNAs coding for all four subunits of the NMDAR in the hippocampus in SPS rats were higher than those in Sham rats during the entire period of extinction training, (4) repeated administration of DCS eliminated the enhanced mRNA levels of NMDARs in the rat hippocampus found 7 days after SPS.

In Experiment 1, SPS rats showed significant impairment of contextual fear extinction as compared with Sham rats. Whereas there was no significant difference in the freezing levels between SPS and Sham rats $24 \mathrm{~h}$ after the fear conditioning, the freezing levels in SPS rats were significantly higher than those in Sham rats after the second extinction training. We recently found no significant difference in locomotor activities between SPS and Sham rats (Imanaka et al, 2006; Takahashi et al, 2006). In addition, we reported that the freezing response in SPS rats is not due to abnormalities of sensory-motor function (Takahashi et al, 2006).

Since an initial report by Walker et al (2002), a growing body of evidence has indicated that DCS administration enhances the extinction of conditioned fear (Ledgerwood et al, 2003, 2004, 2005; Parnas et al, 2005; Woods and Bouton, 2006). In Experiment 2, we observed that repeated DCS administration alleviated the impaired extinction of contextual fear in SPS rats. This effect was also found 7 days after the fifth extinction training. In contrast, the enhanced effect of repeated DCS administration upon extinction was not observed in Sham rats. One possible explanation for this result is that the duration of context exposure (10 $\mathrm{min}$ ) may be longer for Sham rats. Since the freezing levels in Sham rats decreased relatively rapidly, it is possible that we were not able to ascertain the enhanced effect of DCS (ie a floor effect). Another explanation is that, owing to the limitations of our administration procedure, it is possible that each rat failed to consume an adequate amount of Milli-Q water containing DCS.

Our results are generally in agreement with the results of Walker et al (2002) and Ledgerwood et al (2003), in which DCS systemic administration enhanced the extinction of conditioned fear. However, since intraamygdala, as well as systemic administration of DCS enhanced the extinction of conditioned fear, it is postulated that the amygdala plays an important role in the DCS enhancement, even more so than the hippocampus. In this context, it cannot be ruled out that the oral administration of DCS in the present study affected NMDAR function in the amygdala as well as the hippocampus, and consequently alleviated the impaired extinction of contextual fear.

On the other hand, chronic preexposure of DCS has been reported to eliminate effects upon fear extinction (Parnas et al, 2005). Similarly, Quartermain et al (1994) reported that chronic preexposure to DCS for 15 days led to a significant decrease in the effect of DCS upon the maze learning test (Quartermain et al, 1994). The discrepancy observed in the DCS effect upon extinction may be due to differences in experimental procedures. Studies reported by Parnas et al (2005) and Quartermain et al (1994) described the multiple administration of DCS before fear conditioning or the maze learning test, respectively (ie preadministration of DCS). In contrast, in the present study, we administered DCS after fear conditioning but with extinction training. Therefore, it is conceivable that tachyphylaxis or the desensitization of NMDA receptors, as proposed by Parnas et al (2005), may not have occurred in our study.

As mentioned above, the initial level of freezing is also critical in the assessment of contextual fear extinction by DCS. To our knowledge, little has been reported previously concerning the effects of DCS upon fear consolidation. Therefore, in the present study, we examined the possibility that DCS affected the consolidation of original fear. Although we administered DCS after fear conditioning, the results of our study demonstrate that DCS did not affect the consolidation of fear $24 \mathrm{~h}$ after fear conditioning. Subsequently, DCS was found to enhance extinction, rather than consolidation of fear. It is too early to conclude the precise effects of DCS upon fear consolidation from this result; further studies using different experimental paradigms are required.

Studies investigating the clinical use of DCS in the treatment of acrophobia or social anxiety disorder have demonstrated that the acute dosing of DCS enhances fear extinction (Hofmann et al, 2006; Ressler et al, 2004). On the other hand, studies reported by Heresco-Levy et al (2002) did not demonstrate sufficient efficacy for DCS in the treatment of PTSD (Heresco-Levy et al, 2002). This discrepancy could be attributed to procedural difference. Heresco-Levy et al (2002) used daily chronic dosing without exposure therapy, while Hofmann et al (2006) and Ressler et al (2004) used acute dosing with exposure therapy. This, considered collectively with findings from animal studies, leads to the postulation that DCS administration without extinction training cannot facilitate fear extinction.

In Experiment 3, our analyses of NMDAR expression in the hippocampus demonstrated that the levels of all four NMDAR subunits in SPS rats were significantly higher than those in Sham rats before and during the entire period of extinction training. Additionally, in Experiment 4, we also found that the administration of DCS eliminated the enhanced mRNA levels of NR2A and NR2B in SPS rats. It is difficult to explain these results. However, one possible explanation could be that SPS leads to NMDA hypofunction in the hippocampus thereby causing a homeostatic increase in NMDA receptor expression. This would account for the relative reduction in neural plasticity. However, the mechanism of action for DCS would actually be more complicated. DCS, irrespective of its mechanism of involvement in the enhancement of fear extinction, may help to reverse hippocampal plasticity, and thus reverse NMDA compensatory alterations.

Recent studies have indicated that hippocampal neural plasticity is involved in fear extinction (Corcoran et al, 2005; Fischer et al, 2004; Heldt et al, 2007). For example, Corcoran et al (2005) have demonstrated that muscimol inactivation of the dorsal hippocampus reduced the rate of extinction and prevented the context dependency of extinction. Previous studies by this group indicated that 
treating the dorsal hippocampus with muscimol produced selective impairment in the context specificity of extinction (Corcoran and Maren, 2001). In addition, Fischer et al (2004) have shown that structural plasticity involving actin rearrangement within dorsal hippocampus is required for contextual fear extinction. More recently, Heldt et al (2007) demonstrated that the hippocampal expression of BDNF is required for the neural plasticity underlying the acquisition or consolidation of extinction memories.

In our study, we did not clarify the mechanism by which DCS alleviated the impairment of extinction in SPS rats. Although the precise mechanism underlying how DCS facilitates fear extinction remains to be determined, several studies have suggested that DCS activates NMDARmediated signal transduction, thus contributing to the enhancement of new learning (Gabriele and Packard, 2007; Land and Riccio, 1999; Quartermain et al, 1994; Rouaud and Billard, 2003). For example, Rouaud and Billard (2003) showed that DCS facilitated NMDAR-mediated signal transduction and synaptic plasticity in the CA1 field of rat hippocampal slices. Interestingly, Davis and colleagues have recently proposed that the NMDARs involved in extinction may be different from those involved in fear conditioning (eg on different neurons) (Davis et al, 2006). To test this hypothesis, further research is warranted.

The limitations of the present study are as follows. First, we administered DCS to the rats orally in an effort to avoid stress incurred via injection and to mimic clinical use. However, using this methodology it was not possible to accurately confirm the amount of Milli-Q water consumed by each rat daily. Hence, it should be noted that the concentration of DCS could only be assumed to be approximately $15 \mathrm{mg} / \mathrm{kg}$. Second, we did not evaluate changes in the protein level of NMDARs, the extent of NMDAR phosphorylation, or intracellular signal transduction mediated by NMDARs in the hippocampus. Converging evidence has indicated that the phosphorylation of mitogen-activated kinase (MAPK)/extracellular signal-regulated kinase (ERK) in the hippocampus via NMDARs plays an important role in fear extinction (Fischer et al, 2007; Szapiro et al, 2003). Therefore, additional studies using immunoblot or immunohistochemical analyses are necessary to address these issues. Third, it should be noted that because there is enhanced NMDA receptor expression in the hippocampus, this does not mean that the same is happening in other areas of brain. In addition, it is plausible that the enhanced NMDA receptor expression observed in the hippocampus could be a consequence, but not a causative factor, of impaired fear extinction. Finally, we cannot rule out the possibility of the involvement of other brain regions in fear extinction as described above. Numerous studies have suggested that the amygdala and medial prefrontal cortex (mPFC) also play an important role in extinction (Barad et al, 2006; Quirk et al, 2006; SotresBayon et al, 2006). The hippocampus has strong reciprocal connections with the mPFC and the amygdala; these three brain regions seem to interactively coordinate the encoding and expression of fear extinction (Sotres-Bayon et al, 2006).

In summary, SPS rats showed impaired fear extinction in the contextual fear-conditioning paradigm. Furthermore, repeated DCS administration ameliorated the impaired extinction in SPS rats. This is the first study indicating the efficacy of DCS upon fear extinction in a conditioned stress model of PTSD. Although the precise mechanism of fear extinction remains unknown, the results of the present study indicate that NMDAR-mediated signal transduction in the hippocampus may be involved in the pathophysiology of fear extinction. Studies concerning fear extinction using the intrahippocampus infusion of DCS have yet to be performed. Further studies administering DCS into the hippocampus would greatly enhance our understanding of the mechanisms underlying DCS action. Further studies examining both the neural mechanisms underlying the effect of DCS and the efficacy of DCS in the treatment of PTSD are needed to further our understanding of the pathophysiology of PTSD and for the development of novel therapeutic strategies for PTSD.

\section{ACKNOWLEDGEMENTS}

We thank A Matsuki for technical support, and Drs T Takahashi, Y Iwamoto, A Imanaka, S Toki and K Erabi for their helpful advice.

\section{DISCLOSURE/CONFLICT OF INTEREST}

The authors declare that this work was funded by a grantin-aid for general scientific research from the Ministry of Education, Science, and Culture of Japan, a Health Science Research Grant for Research on Brain Science from the Ministry of Health and Welfare of Japan, and a grant from Core Research for Evolutional Science and Technology (CREST) of Japan Science and Technology (JST). The authors also declare that, except for the fund described above, no financial support or compensation has been received from any individual or corporate entity over the past three years for research or professional service and there are no personal financial holdings that could be perceived as constituting a potential conflict of interest.

\section{REFERENCES}

American Psychiatric Association (APA) (1994). Diagnostic and Statistical Manual of Mental Disorders, 4th edn. APA: Washington, DC.

Barad M, Gean PW, Lutz B (2006). The role of the amygdala in the extinction of conditioned fear. Biol Psychiatry 60: 322-328.

Bevilaqua LR, da Silva WN, Medina JH, Izquierdo I, Cammarota M (2005). Extinction and reacquisition of a fear-motivated memory require activity of the Src family of tyrosine kinases in the CA1 region of the hippocampus. Pharmacol Biochem Behav 81: 139-145.

Corcoran KA, Desmond TJ, Frey KA, Maren S (2005). Hippocampal inactivation disrupts the acquisition and contextual encoding of fear extinction. J Neurosci 25: 8978-8987.

Corcoran KA, Maren S (2001). Hippocampal inactivation disrupts contextual retrieval of fear memory after extinction. J Neurosci 21: $1720-1726$.

Davis M, Ressler K, Rothbaum BO, Richardson R (2006). Effects of D-cycloserine on extinction: translation from preclinical to clinical work. Biol Psychiatry 60: 369-375.

Falls WA, Miserendino MJ, Davis M (1992). Extinction of fearpotentiated startle: blockade by infusion of an NMDA antagonist into the amygdala. J Neurosci 12: 854-863. 
Fischer A, Radulovic M, Schrick C, Sananbenesi F, GodovacZimmermann J, Radulovic J (2007). Hippocampal Mek/Erk signaling mediates extinction of contextual freezing behavior. Neurobiol Learn Mem 87: 149-158.

Fischer A, Sananbenesi F, Schrick C, Spiess J, Radulovic J (2004). Distinct roles of hippocampal de novo protein synthesis and actin rearrangement in extinction of contextual fear. $J$ Neurosci 24: 1962-1966.

Gabriele A, Packard MG (2007). D-Cycloserine enhances memory consolidation of hippocampus-dependent latent extinction. Learn Mem 14: 468-471.

Heldt SA, Stanek L, Chhatwal JP, Ressler KJ (2007). Hippocampusspecific deletion of BDNF in adult mice impairs spatial memory and extinction of aversive memories. Mol Psychiatry 12: 656-670.

Heresco-Levy U, Kremer I, Javitt DC, Goichman R, Reshef A, Blanaru $\mathrm{M}$ et al (2002). Pilot-controlled trial of D-cycloserine for the treatment of post-traumatic stress disorder. Int J Neuropsychopharmacol 5: 301-307.

Hofmann SG, Meuret AE, Smits JA, Simon NM, Pollack MH, Eisenmenger $\mathrm{K}$ et al (2006). Augmentation of exposure therapy with D-cycloserine for social anxiety disorder. Arch Gen Psychiatry 63: 298-304.

Imanaka A, Morinobu S, Toki S, Yamawaki S (2006). Importance of early environment in the development of post-traumatic stress disorder-like behaviors. Behav Brain Res 173: 129-137.

Khan S, Liberzon I (2004). Topiramate attenuates exaggerated acoustic startle in an animal model of PTSD. Psychopharmacology (Berl) 172: 225-229.

Land C, Riccio DC (1999). D-Cycloserine: effects on long-term retention of a conditioned response and on memory for contextual attributes. Neurobiol Learn Mem 72: 158-168.

Ledgerwood L, Richardson R, Cranney J (2003). Effects of D-cycloserine on extinction of conditioned freezing. Behav Neurosci 117: 341-349.

Ledgerwood L, Richardson R, Cranney J (2004). D-cycloserine and the facilitation of extinction of conditioned fear: consequences for reinstatement. Behav Neurosci 118: 505-513.

Ledgerwood L, Richardson R, Cranney J (2005). D-cycloserine facilitates extinction of learned fear: effects on reacquisition and generalized extinction. Biol Psychiatry 57: 841-847.

Liberzon I, Krstov M, Young EA (1997). Stress-restress: effects on ACTH and fast feedback. Psychoneuroendocrinology 22: 443-453.

Liberzon I, Lopez JF, Flagel SB, Vazquez DM, Young EA (1999). Differential regulation of hippocampal glucocorticoid receptors mRNA and fast feedback: relevance to post-traumatic stress disorder. J Neuroendocrinol 11: 11-17.

Milad MR, Rauch SL, Pitman RK, Quirk GJ (2006). Fear extinction in rats: implications for human brain imaging and anxiety disorders. Biol Psychol 73: 61-71.

Parnas AS, Weber M, Richardson R (2005). Effects of multiple exposures to D-cycloserine on extinction of conditioned fear in rats. Neurobiol Learn Mem 83: 224-231.
Quartermain D, Mower J, Rafferty MF, Herting RL, Lanthorn TH (1994). Acute but not chronic activation of the NMDA-coupled glycine receptor with $\mathrm{D}$-cycloserine facilitates learning and retention. Eur J Pharmacol 257: 7-12.

Quirk GJ, Garcia R, Gonzalez-Lima F (2006). Prefrontal mechanisms in extinction of conditioned fear. Biol Psychiatry 60: 337-343.

Rauch SL, Shin LM, Phelps EA (2006). Neurocircuitry models of posttraumatic stress disorder and extinction: human neuroimaging research-past, present, and future. Biol Psychiatry 60: 376-382.

Ressler KJ, Rothbaum BO, Tannenbaum L, Anderson P, Graap K, Zimand $\mathrm{E}$ et al (2004). Cognitive enhancers as adjuncts to psychotherapy: use of $\mathrm{D}$-cycloserine in phobic individuals to facilitate extinction of fear. Arch Gen Psychiatry 61: 1136-1144.

Rodrigues SM, Schafe GE, LeDoux JE (2001). Intra-amygdala blockade of the NR2B subunit of the NMDA receptor disrupts the acquisition but not the expression of fear conditioning. J Neurosci 21: 6889-6896.

Rothbaum BO, Davis M (2003). Applying learning principles to the treatment of post-trauma reactions. Ann NY Acad Sci 1008: $112-121$.

Rouaud E, Billard JM (2003). D-cycloserine facilitates synaptic plasticity but impairs glutamatergic neurotransmission in rat hippocampal slices. Br J Pharmacol 140: 1051-1056.

Santini E, Muller RU, Quirk GJ (2001). Consolidation of extinction learning involves transfer from NMDA-independent to NMDAdependent memory. J Neurosci 21: 9009-9017.

Sotres-Bayon F, Bush DE, LeDoux JE (2004). Emotional perseveration: an update on prefrontal-amygdala interactions in fear extinction. Learn Mem 11: 525-535.

Sotres-Bayon F, Cain CK, Ledoux JE (2006). Brain mechanisms of fear extinction: historical perspectives on the contribution of prefrontal cortex. Biol Psychiatry 60: 329-336.

Suenaga T, Morinobu S, Kawano K, Sawada T, Yamawaki S (2004). Influence of immobilization stress on the levels of CaMKII and phospho-CaMKII in the rat hippocampus. Int J Neuropsychopharmacol 7: 299-309.

Szapiro G, Vianna MR, McGaugh JL, Medina JH, Izquierdo I (2003). The role of NMDA glutamate receptors, PKA, MAPK, and CAMKII in the hippocampus in extinction of conditioned fear. Hippocampus 13: 53-58.

Takahashi T, Morinobu S, Iwamoto Y, Yamawaki S (2006). Effect of paroxetine on enhanced contextual fear induced by single prolonged stress in rats. Psychopharmacology (Berl) 189: 165-173.

Walker DL, Ressler KJ, Lu KT, Davis M (2002). Facilitation of conditioned fear extinction by systemic administration or intraamygdala infusions of D-cycloserine as assessed with fearpotentiated startle in rats. J Neurosci 22: 2343-2351.

Woods AM, Bouton ME (2006). D-cycloserine facilitates extinction but does not eliminate renewal of the conditioned emotional response. Behav Neurosci 120: 1159-1162.

Supplementary Information accompanies the paper on the Neuropsychopharmacology website (http://www.nature.com/npp) 